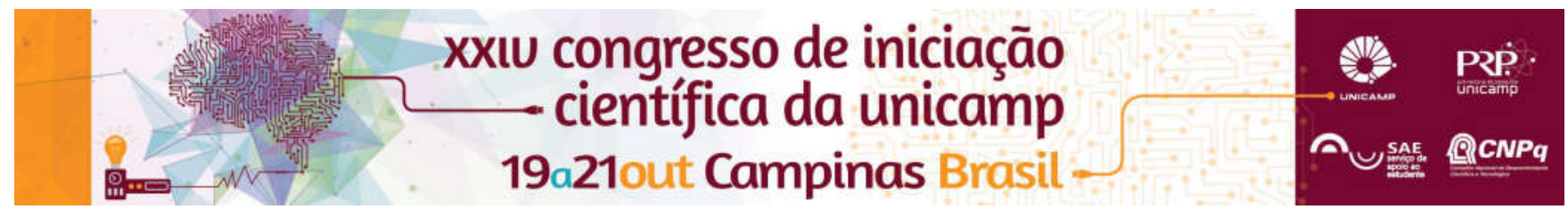

\title{
Study of the Raman enhancement and of local temperature increase probed by molecules adsorbed on Au nanostructures
}

\author{
Thalita P. Damaceno*, Diego P. dos Santos.
}

\begin{abstract}
Surface plasmon resonance of Au nanoparticles causes, among other optical effects, the enhancement of Raman signal known as SERS (surface-enhanced Raman scattering) and the increase of local temperature. In this work, we present an experimental (SERS)/theoretical (Mie theory) study of such effects for cresyl violet (CV) adsorbed on Au nanoparticle clusters.
\end{abstract}

\section{Key words:}

SERS, nanostructures, plasmonics, temperature.

\section{Introduction}

The surface plasmon resonance of metallic nanoparticles allows the amplification of the electric field of the incident radiation on the surface of these nanoparticles when in resonance conditions. This effect is the major contribution to SERS. ${ }^{1}$ Furthermore, the plasmon resonance leads to local temperature increases. ${ }^{2}$

This research project aims at investigating the effect of geometry and agglomeration of $\mathrm{Au}$ nanoparticles as sources of SERS and local temperature enhancements. Once the anti-Stokes to Stokes ratios $\left(I_{A S} / I_{S}\right)$ is dependent on the resonance conditions and on temperature ${ }^{3}$ we performed experimental and theoretical analysis of such quantity as a single parameter to study both effects.

\section{Results and Discussion}

Image 1 presents the experimental $\mathrm{I}_{\mathrm{AS}} / \mathrm{I}_{\mathrm{S}}$ for the band at 590 $\mathrm{cm}^{-1}$ of cresyl violet molecule.

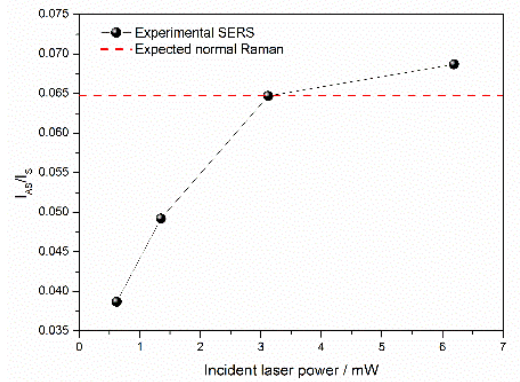

Image 1. $I_{\text {AS }} / I_{S}$ ratio as function of laser power.

Image 1 shows that $I_{A S} / I_{S}$ increases with laser power, an indicative of local temperature increase ${ }^{3}$ and that $I_{A S} / I_{S}$ can be used as probe of local temperature. However, the expected $I_{A S} / I_{S}$ in normal Raman conditions is larger than most of the experimental SERS events, which indicates a contribution from resonance (electric field enhancement) ${ }^{3}$. To account for such experimental result, Figure 2 shows simulations for some of the observed Au aggregates in the scanning electron microscopy (SEM) image.

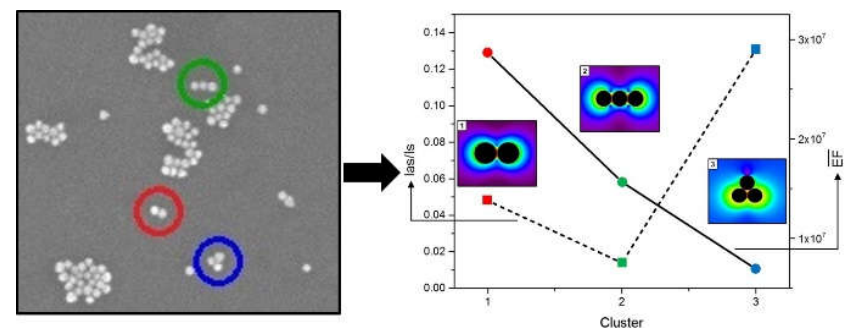

Image 2. $I_{A S} / I_{S}$ ratios and enhancement factor $(E F)$ for the selected clusters in the SEM image.

Image 2 shows that $I_{A S} / I_{S}$ can be very high at room temperature for clusters comprised of nearly touching nanoparticles (cluster $3, \mathrm{I}_{\mathrm{AS}} / \mathrm{I}_{\mathrm{S}}=0.13$ ). Most of the observed clusters have similar configurations (with more particles); however, this cluster presents the smaller contribution to EF (therefore total SERS intensity), which may explain the observed experimental results.

\section{Conclusions}

$\mathrm{I}_{\mathrm{AS}} / \mathrm{IS}_{\mathrm{S}}$ can be a good parameter to investigate the surface plasmon resonance condition (electric field enhancements) and local temperature increases. The low $\mathrm{I}_{\mathrm{AS}} / \mathrm{I}_{\mathrm{S}}$ (in respect to normal Raman) can be explained by electric field resonance conditions on small clusters (favoring the Stokes side). Such clusters dominate the total SERS intensity.

\section{Acknowledgement}

The authors thank CNPq for financial support and IQUNICAMP for infrastructure.

\footnotetext{
${ }^{1}$ Ru, E. C. L.; Etchegoin, P. G. Principles of Surface-Enhanced Raman Spectroscopy And Related Plasmonic Effects; Elsevier, 2008.

${ }^{2}$ dos Santos, D. P.; Temperini, M. L. A.; Brolo, A. G. In Introduction to Plasmonics; Pan Stanford, 2015; pp 275-317.

${ }^{3}$ dos Santos, D. P.; Temperini, M. L. A.; Brolo, A. G. Single-Molecule SurfaceEnhanced (Resonance) Raman Scattering (SE(R)RS) as a Probe for Metal Colloid Aggregation State. J. Phys. Chem DOI: 10.1021/acs.jpcc.6b02400.
} 